\title{
A Split-Luciferase Reporter Recognizing GFP and mCherry Tags to Facilitate Studies of Protein-Protein Interactions
}

\author{
Mehdi Moustaqil ${ }^{1,2, \dagger}$, Akshay Bhumkar ${ }^{1,2,+}$, Laura Gonzalez ${ }^{1,2}$, Lisa Raoul ${ }^{2, \ddagger}$, \\ Dominic J. B. Hunter ${ }^{1}$, Pascal Carrive ${ }^{2}$, Emma Sierecki ${ }^{1,2, *}$ and Yann Gambin ${ }^{1,2, *}$ \\ 1 European Molecular Biology Laboratory Australia (EMBL Australia) Node in Single Molecule Science, \\ Sydney NSW 2031, Australia; m.moustaqil@student.unsw.edu.au (M.M.); a.bhumkar@unsw.edu.au (A.B.); \\ interacteambio@gmail.com (L.G.); d.hunter@imb.uq.edu.au (D.J.B.H.) \\ 2 School of Medical Sciences, The University of New South Wales, Sydney NSW 2031, Australia; \\ lisa.raoul@ens-rennes.fr (L.R.); p.carrive@unsw.edu.au (P.C.) \\ * Correspondence: e.sierecki@unsw.edu.au (E.S.); y.gambin@unsw.edu.au (Y.G.); \\ Tel.: +61-(2)-9385-8933 (E.S. \& Y.G.) \\ + These authors contributed equally. \\ $\ddagger$ Current address: Ecole Normale Superieure de Rennes, 35170 Bruz, France.
}

Received: 31 October 2017; Accepted: 5 December 2017; Published: 11 December 2017

\begin{abstract}
The use of fluorescently-tagged proteins in microscopy has become routine, and anti-GFP (Green fluorescent protein) affinity matrices are increasingly used in proteomics protocols. However, some protein-protein interactions assays, such as protein complementation assays (PCA), require recloning of each protein as a fusion with the different parts of the complementation system. Here we describe a generic system where the complementation is separated from the proteins and can be directly used with fluorescently-tagged proteins. By using nanobodies and performing tests in cell-free expression systems, we accelerated the development of multiple reporters, detecting heterodimers and homodimers or oligomers tagged with GFP or mCherry. We demonstrate that the system can detect interactions at a broad range of concentrations, from low nanomolar up to micromolar.
\end{abstract}

Keywords: protein-protein interaction; split-luciferase; universal reporter; Leishmania tarentolae cell-free

\section{Introduction}

It is currently estimated that over 650,000 protein-protein interactions exist in the human interactome [1] and constant efforts are ever-expanding this number. These interactions are central to cellular functions and are emerging targets for pharmacological intervention when implicated in a particular disease pathway [2]. Multiple in vitro and in vivo methods are reported in the literature to target and study these biomolecular interactions [3-6]. In vitro assays are mostly used for interrogating protein-protein and protein-DNA interactions and their antagonists. Commonly used techniques include variations of enzyme-linked immunosorbent assays (ELISAs) [7], surface plasmon resonance [8], and fluorescence polarization [9], which either require the use of antibodies or purified, often chemically derivatized, proteins. In vivo, powerful methods such as yeast two-hybrid assays [10] have the advantage of speed by eliminating the need for protein purification but can be subject to false positives and false negatives due to the multifactorial nature of signal generation [11]. Strategies to investigate protein-protein interactions (PPI) have become a crucial component in efforts to define the gene function, information flow, and organization of biochemical networks. It is with no 
surprise that in the last decade we have seen the emergence of new and optimized techniques to detect protein-protein interactions, both in vivo and in vitro.

Here we will focus more specifically on Protein Complementation Assays (PCA), also referred to as "split" systems [12]. In these assays, a reporter protein with enzymatic properties is "split" into two inactive fragments. Potential interacting proteins are fused separately with the two fragments. Upon interaction, the two fragments are brought into close proximity and re-assemble spontaneously into the active reporter. The first reconstitution of a functional protein from fragments was demonstrated in 1959 on a ribonuclease [13], but it was only in 1994 that the PCA was developed for PPI by Johnsson and Varshavsky [14]. They demonstrated that an ubiquitin (Ub)-based split-protein sensor could be used to examine the kinetics and equilibrium aspects of a PPI at its natural sites in a living cell. Since then, split-protein pairs have been developed using a variety of scaffolds from ubiquitin, GFP [15] and its variants [16,17], $\beta$-Galactosidase [18], dihydrofolate reductase (DHFR) [19], $\beta$-Lactamase [20], firefly [21], luciferase [22], or, more recently, thymidine kinase [23] and chorismate mutase [24]. These proteins support diverse enzymatic activities that can be varyingly difficult to quantify. The simplest assays are based on fluorescent proteins as detection of the fluorescent signal is straightforward $[25,26]$. On the other hand, luciferase is extensively used in biology to measure gene expression, as luminescence can be easily quantified on plate readers. Assays based on split-luciferases (split-luc) are therefore often chosen due to their ease of use and good sensitivity.

Even though PCA methods are extremely valuable tools for imaging and probing dynamic cellular processes, over the past decade, they have shown certain limitations. Long transfection times and the need to propagate cellular cultures prior to analysis are some of the main limitations in vivo. In vitro, it is mainly the extensive amount of protein purification required [27], associated with the proper folding of the recombinant proteins, that acts as a limiting factor. Last but not least, split-protein assays face other problems such as potential proteolysis of intracellularly-expressed proteins and peptides [28], as well as a lack of control over interfering co-expressed cellular factors.

However, the main limiting factor for PCA development is the need to design a specific reporter for each protein pair that is investigated. The PCA fragments need to be fused directly to the protein of interest, and the number of configurations to explore will rapidly increase the cloning costs $[29,30]$. Indeed, for each protein, one needs to choose which fragment to add, decide on the $\mathrm{N}$-terminal or C-terminal tagging of the protein, and maybe try different linkers, and all these tasks are time-consuming and reduce the number of protein pairs studied. Here we discuss a new universal PPI and protein oligomerisation detector which can be adapted to any GFP- and/or mCherry-tagged protein system. Our system utilizes anti-GFP or anti-Cherry nanobodies fused to the split fragments of the newly-developed small luciferase nanoLuc (nLuc). In this study, we also take advantage of cell-free expression systems which help bypass the tedious protein purification steps allowing for rapid PPI detection [31]. This approach is already commonly used for rapid mapping of PPI [32], and here we have expanded it to address issues specific to a dynamic binary structure. We show that the new system can be successfully used to detect not only PPI and protein oligomerization, but can also be extrapolated as a competitor/inhibitor screen.

\section{Results}

\subsection{Design and Universal Application}

To design our reporter, we took advantage of nLuc, a small and bright luciferase which was systematically engineered from deep-sea luminous shrimp luciferase [33]. This was developed by Promega (Madison, WI, USA) into a protein complementation assay by splitting the full-length nLuc into a large bit (L, $18 \mathrm{kDa})$ and a small bit (S, $1.3 \mathrm{kDa})$.

The traditional split-luciferase assay is designed by fusing the individual bits of split-luciferase (L and S) to the interacting proteins (A and B) (Figure 1A). This involves several rounds of cloning using restriction enzyme digestion and then subsequent ligation which is specific to every protein 
pair that is investigated. To create a generic split-luciferase system which can be used with any GFP- and mCherry-tagged target proteins, we fused the large (L) and small (S) bits of nLuc to the $\mathrm{N}$ - or C-terminus of either the anti-GFP (G) or anti-Cherry (C) nanobody. A gateway site-directed recombination technique was used to create the fusion proteins and these were tagged with $8 \times$ His tag to facilitate purification. Our approach yields eight possible combinations of split-luc nanobody fusion peptide (SLN) which can be used to detect homo- and heterodimers (Figure 1B). These eight SLNs were labelled as GL, LG, CL, LC, GS, SG, CS, and SC, based on the terminal of the nanobody where the nLuc bit is fused. For example, GL is the construct that has an anti-GFP nanobody $(G)$ followed by the large (L) bit of nLuc. SC corresponds to the construct where the small (S) bit of nLuc is followed by the anti-Cherry nanobody (C). The nanobodies and split nLuc bits are separated by a 15 -amino acid flexible linker (Table S1), which allows rotational and directional flexibility for optimal recombination upon successful protein-protein interaction (Figure 1C).
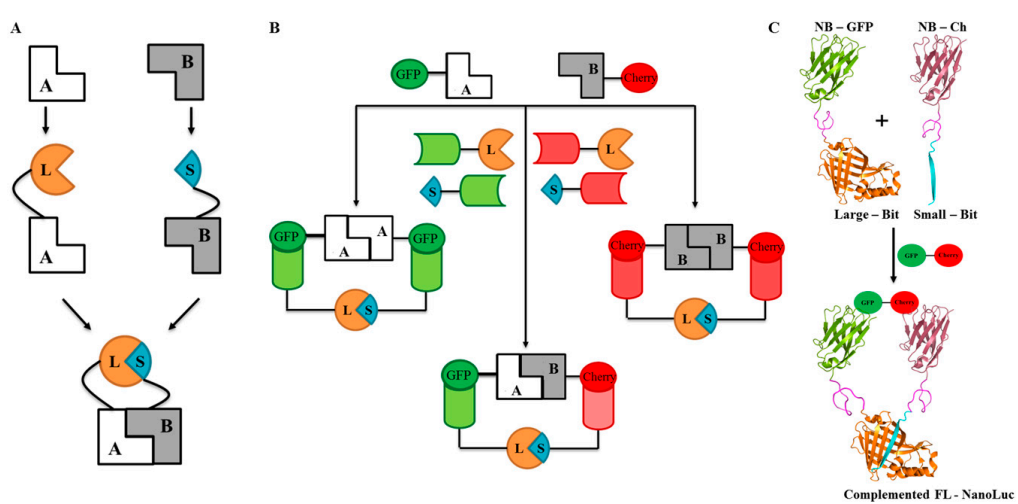

Figure 1. Schematic representations of protein complementation assays. (A): A generic split-luciferase assay is shown where the large bit (orange) (L-residue 1-162) and small bit (blue) (S-residue 163-174) of the split-luciferases (split-luc) (of which neither of them are active individually) are fused to interacting proteins $\mathrm{A}$ and $\mathrm{B}$. Upon successful interaction between $\mathrm{A}$ and $\mathrm{B}$, the $\mathrm{L}$ and $\mathrm{S}$ reconstitute resulting in luminescence [33]; (B): Schematic representation of a universal binary reporter system where the Large bit (L-orange) and small bit (S-blue) of the nLuc are fused with GFP-/mCherry-nanobody (light green and light red respectively) at either the N-/C-terminal. The fused proteins are separated by a flexible linker (GSSGGGGSGGGGSSG). This universal reporter system can detect either protein homodimers for GFP-tagged proteins (green) (eGFP-A:A-sGFP) and mCherry-tagged proteins (mCherry-B:B-mCherry), or heterodimers of GFP- and mCherry-tagged (red) proteins (eGFP-A:B-mCherry); (C): Crystal structure representation of the universal binary reporter split-luc assay of GFP-nanobody, split-luc large bit, and the small bit $[33,34]$. The anti-GFP nanobody (light green) is fused with large bit (orange) of nLuc and the anti-mCherry nanobody (light red) is fused with small bit (blue). This complex when mixed with GFP-mCherry heterodimer (green and red respectively), the binding of anti-GFP and anti-mCherry nanobody to their respective partners facilitates the complementation of full-length nLuc reporter.

The nanobodies have binding affinities in the sub-nanomolar range $(0.49 \mathrm{nM}$ for GFP and $1.4 \mathrm{nM}$ for Cherry), outcompeting the intrinsic affinity of the nLuc subunits $(190 \mu \mathrm{M})$, so we expect that the split-luciferase complementation will not be self-driven but will depend on PPI [33-35]. To validate this, every luciferase assay is carried out using cell-free lysate alone (Figure S1) as negative control. At all concentrations of SLNs, luminescence was at background level suggesting that self-recombination of the bits was negligible.

\subsection{Proof of Principle and Calibration of the PPI Assay}

To test the ability of the system to detect protein homo- and hetero-dimers, eGFP-sGFP, mCherry-sGFP, and mCherry-mCherry tandems were used as models and tested against all 8 potential pairs of SLNs in an $8 \times 8$ matrix. The targets and the SLNs were both expressed in Leishmania cell-free expression system, using Leishmania tarentolae extracts (LTE) and were 10-fold diluted post-expression 
before using them for the PCA. Each of the assay components were mixed in the ratio of 1:1:1 and incubated for $10 \mathrm{~min}$ at $23^{\circ} \mathrm{C}$ before adding the nLuc substrate.

In each case (eGFP-sGFP, eGFP-mCherry, or mCherry-mCherry), signals were detected with excellent signal-to-background ratios and with at least one configuration of the reporter. As shown in Figure 2, the best hits were GL/GS (Figure 2A, eGFP-sGFP homodimer), LC/GS (Figure 2B, mCherry-sGFP heterodimer), and CL/CS (Figure 2C, mCherry-mCherry homodimer). For all $8 \times 8$ matrices, the large-large and small-small self-associations of SLNs gave the lowest signal. The best hits from our $8 \times 8$ matrices were more extensively tested against all target proteins. To validate our data in all cases, we used other constructs as negative controls. For example, in the eGFP-sGFP detection assay, (Figure 2D) both mCherry-mCherry and mCherry-sGFP acted as background controls. At all dilutions of the SLN, each individual nLuc pair showed maximum reactivity to its target, with a signal-to-background ratio of at least 3 , and at best, more than 30 (Figure 2D-F).

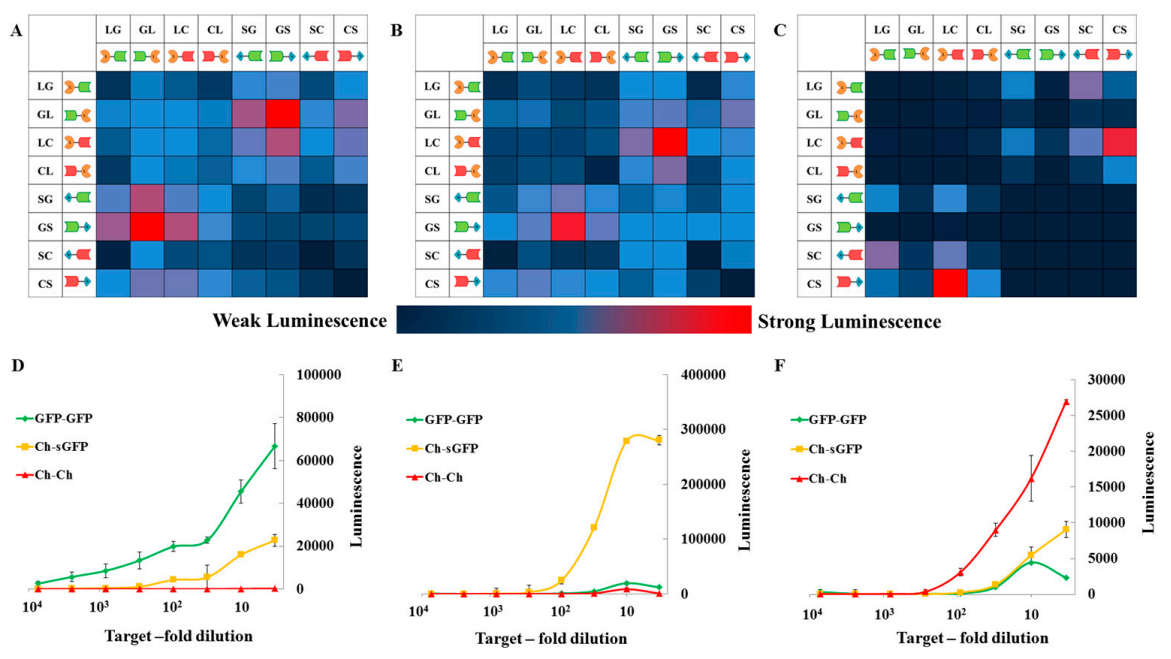

Figure 2. Heat-map and graphical representations of the selectivity of specific split-luc nanobody fusion peptide (SLN) bit pairs. (A-C): Heat-map representations of luminescence observed with $8 \times 8$ matrices of SLN bits as tested on eGFP-sGFP homodimer (A), mCherry-sGFP heterodimer (B), and $\mathrm{mCherry-mCherry} \mathrm{homodimer}(\mathbf{C})$. The heat-map represents higher luminescence signals in red with weaker luminescence transiting towards the blue. As expected, SLNs self-binding give the least signal of all the targets; (D-F): Graphical representation of luminescence signals for individual targets plotted against the serial dilution of SLNs. The assay was run using the best SLN pairs as observed from the $8 \times 8$ matrix. The SLN pairs used for the target proteins were: GL-GS (D), LC-GS (E), and CL-CS (F). Each of the pairs were tested on all the targets and their signals are plotted in individual graphs as eGFP-sGFP (green), mCherry-sGFP (orange), and mCherry-mCherry (red). The average \pm SEM for at least three experiments is plotted. LG: Large bit-anti-GFP, GL: anti-GFP-Large bit, LC: Large bit-anti-Cherry, CL: anti-Cherry-Large bit, SG: small bit-anti-GFP, GS: anti-GFP-Small bit, SC: Small bit-anti-Cherry, CS: anti-Cherry-Small bit.

The resulting matrices also showed that a split-luciferase pair in one orientation can perform very much better (sometimes more than 10-fold) than in other conformations (e.g., in comparing GL/GS and LG/GS (Figure 2A)). Despite the fact that the reporters were designed to have a flexible linker separating the Luc bits from the nanobody, the sheer size of the nanobody and the interacting GFP- and/or mCherry-fused proteins could be a limiting factor. The reporter may be able to complement in only one orientation depending on the size of the interacting protein pair and its mode of binding. From this initial screen, two homodimer reporters (GL/GS and LC/CS for GFP- and mCherry-homodimers respectively) and one heterodimer reporter (LC/GS) were selected.

One limitation of the cell-free expression system that we use is that the levels of expression can be low (in general 1-2 $\mu \mathrm{M}$ and sometimes 10-times lower, especially when proteins are coexpressed [36]). 
This feature can be beneficial as the concentrations are often at physiological levels, suggesting that the detected interactions could be relevant and specific. However, to detect these interactions and be useful in cellulo, the PPI-detection method has to be highly sensitive. From our initial screen, we knew that our system was working in the cell-free extracts. Thus, we wanted to see how the system behaves at different target and SLN concentrations. This was established for the detection of mCherry-sGFP tandems by the LC/GS reporter. Serial dilutions of the target (mCherry-sGFP) and the reporter (LC/GC) were used corresponding to concentration ranges from 5 to $250 \mathrm{nM}$ and around 0.2 to $1000 \mathrm{nM}$, respectively. At the "normal" concentrations of SLN (estimated at $1 \mu \mathrm{M}$ ), the signal decreased as mCherry-sGFP was diluted. Compared to a "lysate only" control, the presence of mCherry-GFP could be satisfyingly detected above $10 \mathrm{nM}$ (Figure 3A). However, when both the SNLs and the target concentrations were modified, the luminescence maxima shifted with the SNL dilution (Figure 3A,B). A significant signal could still be detected with as low as $10^{5}$-fold dilution of SNL (Figure 3D). This can be rationalized: the signal follows a "hook" pattern wherein maximum signal is detected when the SLN bits and the target proteins are in a 1:1 ratio (Figure 3C). At high concentrations of target proteins, the two parts of the split system may distribute on different tandems and are unable to recombine. With the dilution of the targets, as the target-to-SLN ratio reaches 1:1, it generates maximum signal, as seen in our assays. With further dilutions, the reduction in the number of targets results in loss of signal. It means that, by diluting the SLNs (i.e., reporters), we can shift the optimal range of detection for the interacting protein pair using self-calibration of the system. This means that we can still detect sub-nanomolar concentrations of the target (Figure 3D). Based on the protein concentration, our assay can detect interacting protein pairs (mCherry-sGFP in this case) at $1 \mathrm{nM}$, diluting the SLN in the high pico-molar range with a signal-to-background ratio greater than 5 (Figure 3D). Therefore, the assay should be able to work to detect PPI even with low protein expression.
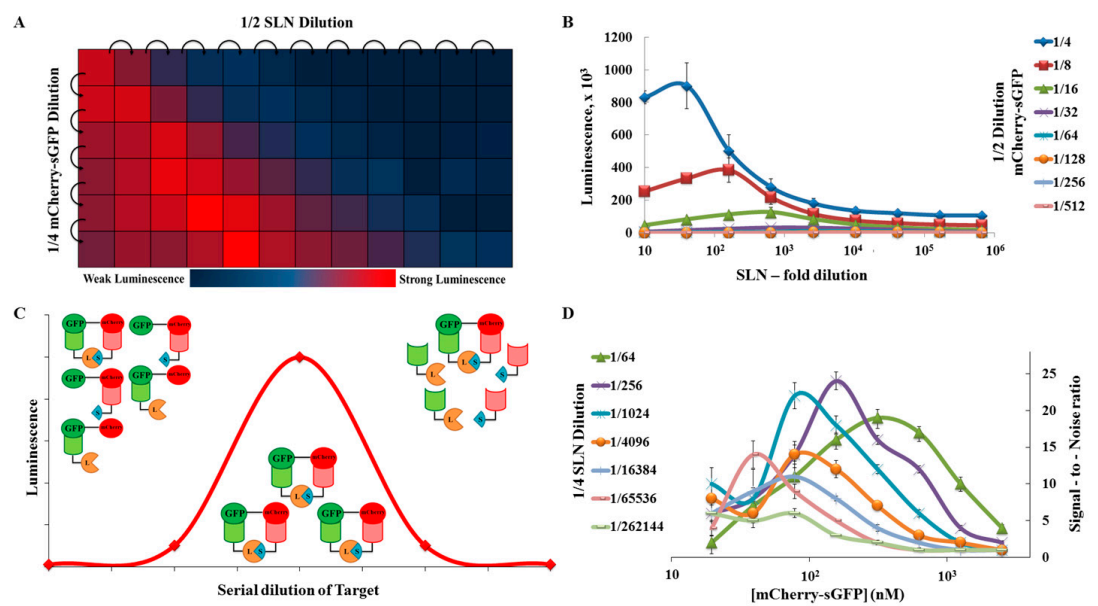

Figure 3. Detection limit studies using a direct approach. (A): Heat-map of the 'hook' effect; the 'hook' effect transition is seen when we plot the luminescence values of SLN and target dilution as a heat-map. Here the highest luminescence is plotted in red and weaker luminescence transits towards blue; (B): Graphical representation of the 'hook' effect as seen with the mCherry-sGFP tandem serial dilution of the LC-GS SLN pair. We see a shift in the 'hook' effect with increasing dilution as the 1:1 ratio of target to SLN shifts to the right; (C): Representation of the 'hook' effect as seen in the split-luc assay. With serial dilution of the target, we see less luminescence when the SLN concentration is less than the target or vice-versa. However, we reach the maxima when the proportion of the SLN and the target proteins reach a 1:1 ratio, giving the highest luminescence; (D): Graphical representation of the signal/noise ratio from the data obtained for graph 3B. The data was plotted by taking the ratio of the luminescence for the target protein and the luminescence observed for the lysate background at the same concentration of SLN. In this case too, we see a 'hook' effect that moves with changes in the concentrations of target and reporter. 
All the previous tests were performed using fused peptides. To evaluate how the system performed on "real" proteins pairs or oligomers, known PPI were selected for each reporter: eGFP-foldon [37] (homotrimer of GFP), sGFP-MyD88 [38], the cMyc(b/HLH/Zip)-HDAC3 [39] pair (heterodimer of sGFP-mCherry), mCherry-SOX9 [40] (mCherry homodimer), and mCherry-Cav1 [32] (Figure 4). For detecting these interacting proteins, the targets and SLNs were separately expressed in Leishmania cell-free lysate and the targets were serially diluted three-fold before adding them to the assay (detailed experiment outlined in the material and methods section). To test the background luminescence originating from the SLN self-interaction, non-expressing Leishmania cell-free lysate was used and it too was serially diluted three-fold before adding it to the assay. In every case, we were able to detect a luminescence signal with a signal-to-background ratio of at least 5 (mCherry-SOX9) and at best 60 (sGFP-MyD88). To establish a non-interacting pair as a negative control, along with cMyc-HDAC3, cMyc-SMAD2 was used. The luminescence from the cMyc-SMAD2 PPI pair was as low as background levels typically measured for a non-expressing LTE control.

A

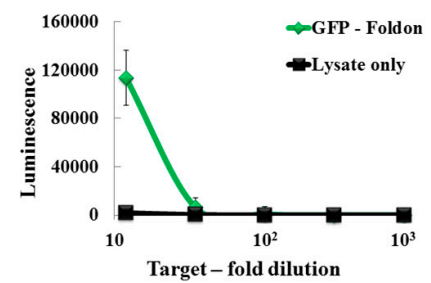

D

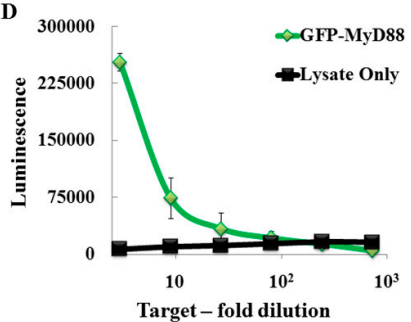

B

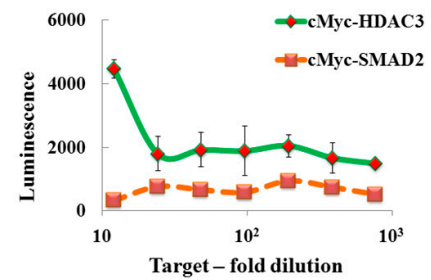

$\mathbf{E}$

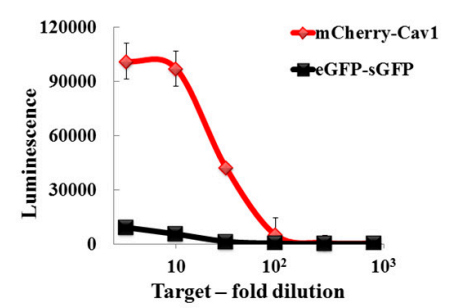

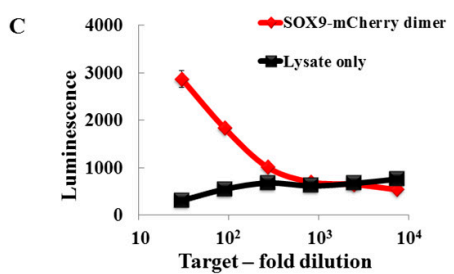

$\mathbf{F}$

\begin{tabular}{|c|c|c|}
\hline Panel & $\begin{array}{c}\text { Protein } \\
\text { (homodimer/heterodimer) }\end{array}$ & $\begin{array}{c}\text { Size of } \\
\text { complex }\end{array}$ \\
\hline A & GFP - Fold-on & 3 \\
\hline B & cMyc-HDAC3 & $1: 1$ \\
\hline C & SOX9 - mCherry & 2 \\
\hline D & GFP - MyD88 & Oligomeric \\
\hline E & mCherry - Cavl & $\sim 100$ \\
\hline
\end{tabular}

Figure 4. Applying the split-luc reporter to PPI and self-dimerising/oligomerising proteins. (A): Graphical representation of split-luc assays performed on the sGFP-Foldon protein. sGFP-Foldon is a trimer wherein GFP trimerizes post-expression due to the foldon domain. We could detect a high luminescence signal over the background for sGFP-Foldon using the GL-GS SLN pair; (B): Graphical representation of split-luc assays performed on the cMyc (b/HLH/Zip)-HDAC3 heterodimer. For this assay, cMyc and HDAC3 were co-expressed in Leishmania cell-free lysate and their serial dilutions were used in the split-luc assay using the LC-GS SLN pair. As a negative control, the non-interacting protein pair cMyc-SMAD2 (dotted line) was used; (C): Graphical representation of split-luc assays performed on the SOX9-mCherry homodimer. The SLN pair of CL-CS was used for this assay with a lysate-only control used to detect background luminescence; (D): Graphical representation of split-luc assays performed on MyD88 using the GL-GS SLN pair; (E): Graphical representation of split-luc assay performed on mCherry-Cav1 using the LC-CFRS SLN pair. As a measure of background luminescence, the eGFP-sGFP homodimer was used; (F): Expected stoichiometry of each target [32,37,38,41,42]. The accession numbers of all the genes used are mentioned in Table S2.

\subsection{Application to Measure PPI Disruption}

Along with standard PPI detection, we wanted to test if the split-luc system could be modified to screen for PPI inhibitors. First however, we determined the characteristics of the assay by creating a competition between eGFP-sGFP (target) and mCherry-sGFP (competitor). This is not an inhibition assay but rather, it mimics a "cold" probe assay (Figure 5A). The mCherry-sGFP competitor, SLNs, and eGFP-sGFP target were expressed in Leishmania cell-free lysate. A single SLN concentration was chosen for this study which would give us a detectable range of signal-to-background ratio 
while not being a rate-limiting factor (in practice 20-fold dilution for an estimated concentration of $50 \mathrm{nM}$ was used). Post-dilution, the SLNs were pre-incubated for $10 \mathrm{~min}$ at $23^{\circ} \mathrm{C}$ with varying concentrations of mCherry-sGFP before adding the desired concentration of the eGFP-sGFP target to the assay. For all eGFP-sGFP concentrations tested, we saw a decrease in luminescence with increasing concentrations of the mCherry-sGFP tandem. The decrease in luminescence could be detected for competitor (mCherry-sGFP) concentrations of as low as $6 \mathrm{nM}$ (Figure 5B). In our case, the assay responded correctly for target (eGFP-sGFP) concentrations between 0.8 and $12 \mathrm{nM}$ (i.e., $1 / 640$ dilution and $1 / 40$ dilution). In this range, the inhibition curves, when normalized against their respective maxima, showed a perfect overlay, suggesting that the mCherry-sGFP inhibition was independent of eGFP-sGFP concentration (Figure 5C) as it should be since we are testing the binding of the anti-GFP nanobody to its substrate. Importantly, this means that in the case of direct detection described above, the competition assay can be performed at different concentrations of target without affecting the results.

$\mathbf{A}$
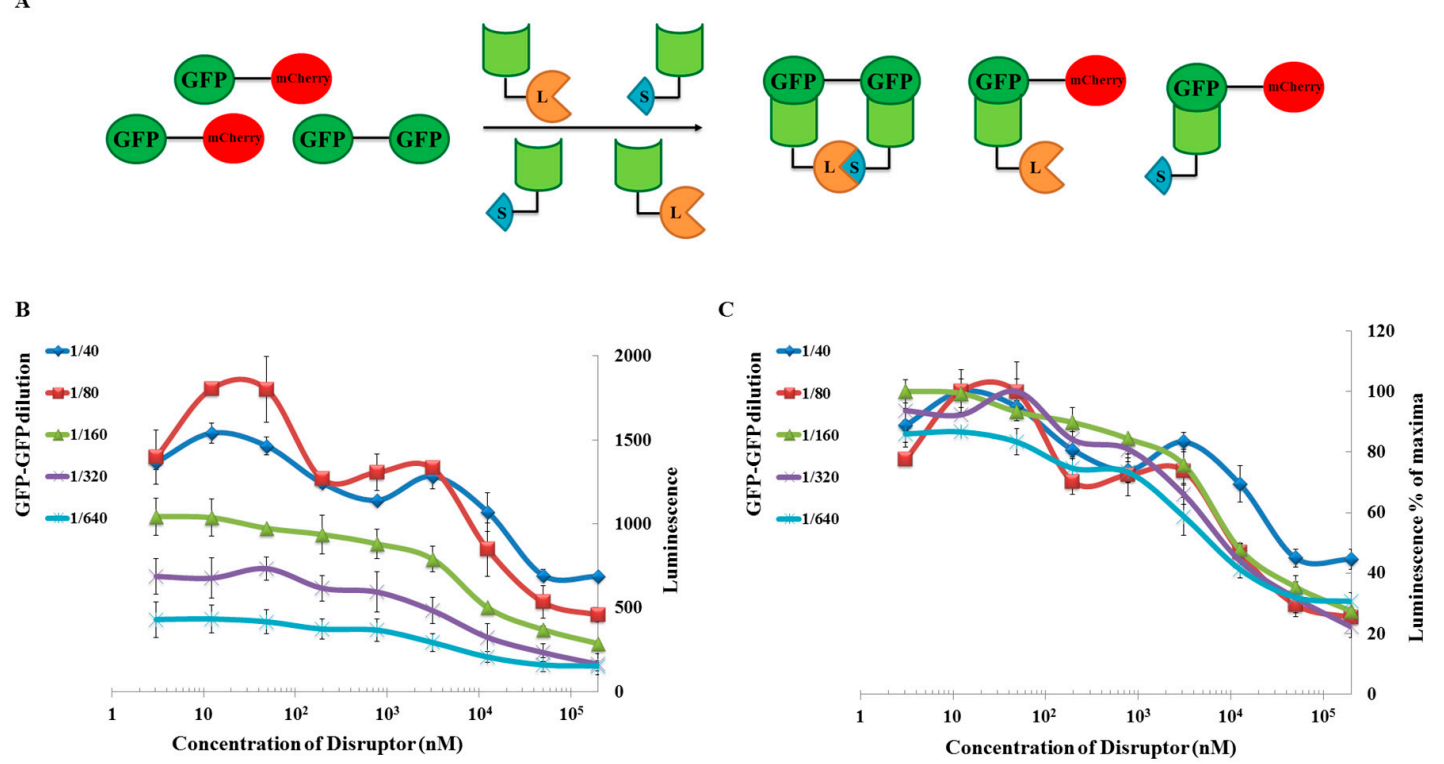

Figure 5. Detection limit studies using a competition approach. (A): Schematic representation of the indirect approach that was used to study the detection limit of the split-luc assay. For the assay, along with the SLN bits and the target protein, we expressed the competitor in a Leishmania cell-free system, and this was used for our assays. The competitor was incubated with SLN bits allowing it bind to the nanobody before adding the target protein and the substrate; (B): Graphical representation of luminescence as a function of competitor concentration; (C): Normalized data-set from the data obtained for graph 5B. The data was normalized against the maximum luminescence obtained for each target concentration and plotted against the competitor concentration. The data-set for each target concentration seems to overlap, suggesting that the inhibition curves obtained were independent of target protein concentration.

Competition between proteins for binding can be used to evaluate the apparent $K_{D}$ of a protein pair. Here we used it to measure the well-described interaction between $\mathrm{cMyc}(\mathrm{b} / \mathrm{HLH} / \mathrm{Zip})$ and MaxZ (b/HLH/Zip) [41]. First, we verified that the protein pair could be detected by our assay. Indeed, when cMyc-mCherry and MaxZ-sGFP were co-expressed in LTE and then added to the LC/GS pair of SLNs, a luminescence signal could be detected well above the background level obtained with lysate only (Figure $6 \mathrm{~A}$, black line). When $25 \mu \mathrm{M}$ of purified MaxZ was added to the assay, a reduction of the luminescence signal was observed (Figure 6A, dotted line). Titration of purified MaxZ into the assay yielded a relationship between luminescence and concentration that could be fitted to reveal the apparent $K_{D}$ of the interaction $\left(K_{D}=1.02 \mu \mathrm{M}, r^{2}=0.8 \%\right.$ ) (Figure $\left.6 \mathrm{~B}\right)$. This value is in good agreement 
with the reported literature where cMyc-MaxZ $K_{D}$ is reported to be in the range of $0.3-76 \mu \mathrm{M}$ and also corresponds to the $K_{D}$ value $\left(K_{D}=3.2 \mu \mathrm{M}\right)$ we obtained using Isothermal Titration Calorimery (ITC) (Figure 6C) [43,44]. Therefore, our generic assay can be adapted to measure affinity constants of protein pairs.
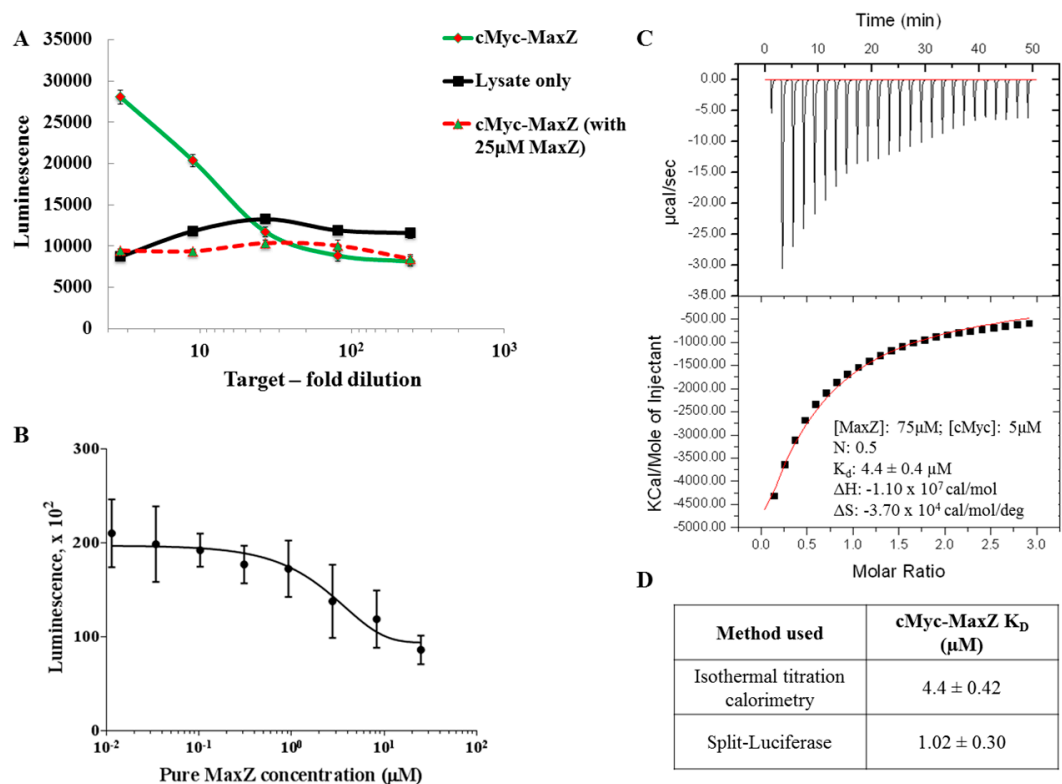

Figure 6. Using split-luciferase assay to determine apparent $K_{D}$ : (A): Graphical representation of split-luc assays performed on the cMyc-MaxZ PPI pair. Here, the non-expressing Leishmania tarentolae extract, (LTE) acts as a negative control (in black). To test if the PPI can be disrupted by pure MaxZ, we used $25 \mu \mathrm{M}$ MaxZ in conjugation with the PPI pair in the split-luc assay. Here, we do see a reduction in luminescence to the background level, due to presence of pure MaxZ; (B): Binding studies of cMyc-MaxZ using purified MaxZ as a PPI disruptor; (C): iTC titration curve for cMyc-MaxZ titration where $75 \mu \mathrm{M}$ MaxZ was titrated in $5 \mu \mathrm{M}$ cMyc and 'one set of site' fitting was used to fit the data to get a $K_{D}$ of $4.4 \mu \mathrm{M}$; (D): Table comparing the binding constants for cMyc-MaxZ using iTC and split-Luc assay.

\section{Discussion}

Genetically-encoded fluorophores are now commonly used in fluorescence microscopy but can also act as purification tags [43-45], folding/solubility markers [46,47], or be developed into functional assays [48-50]. To be able to study PPI and protein oligomerisation using constructs already in use, we created a generic luminescent PCA reporter that can recognize GFP- or mCherry-tagged proteins. Our in-house developed Leishmania cell-free expression system allows us to quickly assess the performances of the assay in multiple configurations. The strong affinity of the GFP and mCherry nanobodies helps push detection limits to lower than has been reported [33]. Owing to its design, by changing the concentration of the reporter, this assay can be applied to an extensive range of target concentrations, from a few micromoles down to nanomolar range, without losing efficacy. This implies that the same assay can be used with similar efficacy on proteins from different sources (from concentrated purified recombinant proteins to proteins in cell extracts at physiological levels) without the need to extensively modify the procedure, and can probably be readily implemented using constructs developed for other uses. Even though the assay should work with higher concentrations of proteins, it is always better to use a more dilute preparation in order to avoid unspecific aggregation of the proteins themselves. The monomeric GFP and Cherry tags were designed to minimize self-association compared to other fluorescent proteins that could form tetrameric assemblies. Our previous experiments testing GFP- and mCherry-tagged proteins 
using single molecule fluorescence detection support our claim that we do not see any endogenous oligomerization associated with fluorescence tags [32,36,38,42,51]. Still, as the split-nLuc assay is very sensitive, the self-association of the fluorescent tags could create false-positive signals and would need to be taken into account at high protein concentrations. Diluting the targets also means that the SLNs can be used at a lower concentration, preventing untriggered self-association and decreasing background fluorescence.

From a pharmaceutical point of view, this can be very valuable. PPI are becoming a very important class of drug targets. When crucial PPI become deregulated, cellular processes are altered; screening for regulated PPI and understanding their role in health and disease [52] is therefore attractive. PPI detection and PPI disruption assays are important in almost all steps of drug discovery, including target identification, assay development, hit identification-validation, lead optimization, and preclinical validation. For example, studying the interactome of a key cellular protein can lead to target identification, disruption of specific PPI in vitro, and will provide the lead compound. On the other hand, in vivo validation of drug effects can require monitoring the loss of an interaction in an organism. Our generic assay could evolve to be very valuable in this perspective as it is able to test different protein pairs without rounds of cloning; it can also be used from cell extracts or purified proteins. It could allow for sensitive and kinetically robust measurements of PPI, and with further developments, be adapted to high throughput applications. So far, we are expressing the SNLs in LTE for each experiment but this is a source of variability. Using purified recombinant SNLs should not only reduce day-to-day variability, but we also expect it to further reduce the background fluorescence, ultimately increasing the signal-to-background ratio of the assay.

The most valuable aspect of our assay is its ability to efficiently detect protein oligomers and aggregates. Protein aggregation is now recognized as a defining component of numerous human disease states including, Alzheimer's (A $\beta$ protein), Huntington's (huntingtin protein), and Parkinson's ( $\alpha$-synuclein protein) [53]. A generic reporter should be able to detect both PPI and self-oligomers. Here, by changing the SLN pair used for the assay, we can adapt the assay to detect PPI (LC/GS) or GFP- or mCherry-tagged protein aggregates (GL/GS and CL/CS) (Figure 4). The signal generated by the presence of oligomers/small aggregates is often larger than the one corresponding to a simple PPI (compare A and B, Figure 4) and can serve as a quick "diagnostic" of protein aggregation. Importantly, when using the PPI reporter (Cherry/GFP recognized by the LC/GS pair respectively), one will only focus on the PPI and ignore the individual GFP/ Cherry oligomers. The parallel use of the three reporters in the same assay format gives a complete picture of the dynamics of the interaction. The self-assembly reporter can provide a simple assay for identifying compounds capable of modulating the aggregation process.

\section{Materials and Methods}

\subsection{Gateway Cloning and Protein Expression}

The gene sequences for the large and the small bits of the split-luc system were obtained from the Promega Nano-Glo ${ }^{\circledR}$ Luciferase Assay System (Madison, WI, USA). ORFs were generated by adding the GFP/mCherry nanobody sequences to either N-/C-terminal of the luc bits, with a 15-amino acid flexible GS linker separating the nanobody and the luc bit. These ORFs were ordered as G-Blocks from IDT and were cloned into the Gateway pDONR221 vector using Gateway ${ }^{\mathrm{TM}}$ BP Clonase ${ }^{\mathrm{TM}}$ II from ThermoFisher Scientific (Waltham, MA, USA). Subsequently, the resulting entry vectors were recombined with the cell-free expression-specific Gateway ${ }^{\mathrm{TM}}$ destination vectors (pCellFree5795) using Gateway $^{\mathrm{TM}}$ LR Clonase ${ }^{\mathrm{TM}}$ II from ThermoFisher Scientific. The destination vectors chosen did not have any intrinsic fluorophore, but had an $\mathrm{N}$-terminal $8 \times$ HIS tag to facilitate purification. The resulting clones were then transformed, colony picked from $\alpha$-select competent cells, and the plasmid DNA was extracted using ZymoPURE ${ }^{\mathrm{TM}}$ Plasmid Midiprep Kit (Irvine, CA, USA). For the cell-free expression of these proteins, the plasmid DNA with the ORFs was added to Leishmania tarentolae cell-free lysate 
(supplemented with feed solution and $\mathrm{Mg}^{2+}$ ) in a ratio of $1: 9[32,51,54,55]$. The protein was allowed to express for two and half hours at $27^{\circ} \mathrm{C}$, before using it for downstream processing.

\subsection{Split-Luciferase Assay for Direct Detection Studies}

The SLNs and the target protein were expressed individually in LTE by adding $1 \mu \mathrm{L}$ of plasmid DNA to $9 \mu \mathrm{L}$ of LTE. The expressed SLNs or the target proteins were serially diluted in buffer A ( $25 \mathrm{mM}$ HEPES $\mathrm{pH} 7.4,50 \mathrm{mM} \mathrm{NaCl}$ ), depending on the assay. For the direct binding assay, each SLN from a large and small bit pair was mixed with target protein in a 1:1:1 ratio $(2 \mu \mathrm{L}: 2 \mu \mathrm{L}: 2 \mu \mathrm{L})$. This mixture was incubated for $10 \mathrm{~min}$ at $23^{\circ} \mathrm{C}$ and $6 \mu \mathrm{L}$ of luciferase substrate (after diluting it as per the manufacturer's recommendation) was added to the mix. The final mixture of SLNs, target protein, and luciferase substrate was incubated for another $10 \mathrm{~min}$ at $23^{\circ} \mathrm{C}$ before reading the luminescence on a PerkinElmer EnSpire plate reader (PerkinElmer, Waltham, MA, USA), using the manufacturer's recommended settings for luminescence assays ( $0.1 \mathrm{~s}$ read per well).

\subsection{Split-Luciferase Assay for Indirect Detection Studies}

SLNs and target proteins (eGFP-sGFP) were expressed individually in LTE by adding $1 \mu \mathrm{L}$ of plasmid DNA to $9 \mu \mathrm{L}$ of LTE. The competitor (mCherry-sGFP) was also expressed in LTE and it was serially diluted in buffer A and mixed in a 1:1:1 ratio with the SLN pair. This mixture was incubated for $20 \mathrm{~min}$ at $23^{\circ} \mathrm{C}$ before adding $2 \mu \mathrm{L}$ of target protein to the mix. To initiate the luciferase reaction, $6 \mu \mathrm{L}$ of luciferase substrate (after diluting it as per the manufacturer's recommendations) was added to the mix and luminescence was read on a PerkinElmer EnSpire plate reader after 10 min of incubation at $23{ }^{\circ} \mathrm{C}$.

\subsection{Split-Luciferase Assay for Apparent $K_{D}$ Calculation}

To detect the apparent $K_{D}$ of the cMyc-MaxZ interaction, MaxZ was purified as per mentioned in Park et al. [56]. For the split-luc assay, MaxZ-sGFP, cMyc-mCherry, and the SLNs (LC and GS) were expressed in LTE (as mentioned above). For the protein pair, cMyc and MaxZ single expressions were mixed in a 2:1 ratio after $10 \mathrm{~min}$ of expression and the mixture was expressed further for $2 \mathrm{~h}$ at $23^{\circ} \mathrm{C}$ before using it for the assay. The SLNs were serially diluted three-fold and were mixed at different concentrations (serial dilutions from 25 to $10 \mathrm{nM}$ ) of purified MaxZ. This mixture was incubated for $20 \mathrm{~min}$ at $23^{\circ} \mathrm{C}$ before adding the protein pairs MaxZ-sGFP and cMyc-mCherry in equal ratios. Finally, luciferase substrate was added to initiate the reaction and luminescence was read on a plate reader after $10 \mathrm{~min}$ incubation at $23^{\circ} \mathrm{C}$. To calculate the $K_{D}$ of the binding, the curves were fit using the one-phase exponential decay equation $\left(\mathrm{Y}=(\mathrm{Y} 0-\right.$ Plateau $) \cdot \exp \left(-K_{D} \cdot \mathrm{X}\right)+$ Plateau $)$ in $\mathrm{GraphPad}^{\circledR}$ Prism.

For measurement of cMyc-MaxZ $K_{D}$ using iTC, both the proteins were purified as per mentioned in Park. et al. [56]. These proteins were dialyzed in Buffer A using GE Healthcare Sephadex G-25 in PD-10 Desalting Columns and were further concentrated to the desired concentration using Merck Amicon ${ }^{\circledR}$ (Kenilworth, NJ, USA) 10 kDa cut-off concentrators. GE Healthcare iTC200 (GE Healthcare, Chicago, IL, USA) was used for our studies wherein $75 \mu \mathrm{M}$ MaxZ was titrated in $5 \mu \mathrm{M}$ cMyc using multi-injection mode (20 injections of $2 \mu \mathrm{L}$ each). The data was then analyzed using Origin ${ }^{\circledR}$ software (Version 5.0, GE Healthcare, Chicago, IL, USA) and the curve was fit for the "one set of sites" equation giving the cMyc-MaxZ $K_{D}$ of $4.4 \mu \mathrm{M}$ [57].

\subsection{Split Luciferase Assay for PPI, Homodimerisation, and Oligomerisation Detection}

To study the PPI using split-luc assays, the interacting pairs of GFP- and mCherry-tagged proteins were individually expressed in LTE for $10 \mathrm{~min}$ (as per mentioned above). These protein pairs were subsequently co-expressed by mixing GFP-expressing LTE with mCherry-expressing LTE in a ratio of $1: 2$ and the mixture was incubated for another $2 \mathrm{~h}$ at $27^{\circ} \mathrm{C}$. In case of homodimerising and oligomerising proteins, the individual GFP-/mCherry-tagged proteins were expressed in LTE (as mentioned above) before being used for the split-luc assay. To facilitate better detection and 
get higher signal-to-background ratios, we developed a longer linker to study mCherry-tagged proteins. This linker was made by combining flexible and rigid linkers (Table S1). This linker was used in conjugation with anti-mCherry nanobodies and small bits from nLuc to form the CFRS SLN (anti-mCherry-Flexible-rigid linker-small bit). The SLN pair LC/CFRS was then used as an mCherry-oligomer detector. The PPI pairs, homodimers, and oligomers were treated like target protein in the split-luc assay and the resulting luminescence was read on a plate reader after $10 \mathrm{~min}$ incubation at $23^{\circ} \mathrm{C}$.

To detect the background luminescence, the non-expressing LTE serial dilution or single dilution was mixed with an equal volume of SLN pairs. This mixture was subject to the same treatment as the target protein and the resulting luminescence was read on a plate reader.

\section{Conclusions}

In conclusion, our generic split-luciferase reporter is designed for the rapid investigation of PPI as well as low limit detection of protein aggregation. Benefiting from the small size and bright luminescence of nLuc, it allows for detection at low concentrations with minimal interference coming from self-association. Notably, conjugating this system with a Leishmania cell-free expression system sidesteps many of the limitations of comparable PCA routinely used in protein analysis. Our assay is distinct from similar PCAs as it can be adapted to any GFP-/mCherry-tagged system and optimized for high throughput screening. Our analysis indicates that this method fulfils the general expectations of a complementation reporter in vitro, with biomolecular parameters generally suited for accurate detection of PPI. In addition to this, in detecting lower levels of protein aggregation, we open up the ability to generate potential pharmacological outcomes. Specifically, this assay can reveal drug potency for induced protein interactions by disrupting the luminescence signal and can also measure the efficiency of drugs $\left(\mathrm{IC}_{50}\right)$. Moreover, with the aptitude of this generic binary reporter, we believe that this luminescence system will provide additional applications yet to be realized. A faster and better understanding of the underlying mechanism of cellular signaling, protein interaction networks, and drug interactions will help improve therapeutic interventions.

Supplementary Materials: Supplementary materials can be found at www.mdpi.com/1422-0067/18/12/2681/s1.

Acknowledgments: This work was supported by grants from the National Health and Medical Research Council of Australia (project grants APP1100771 to Yann Gambin and Emma Sierecki, APP1107643 to Emma Sierecki). Yann Gambin was supported by an Australian Research Council Future Fellowship (FT110100478) during this project.

Author Contributions: Mehdi Moustaqil and Akshay Bhumkar designed the constructs and performed ro directed the experiments and wrote the manuscript. Laura Gonzalez and Lisa Raoul performed experiments. Dominic J. B. Hunter prepared the cell-free extracts used in the study. Pascal Carrive participated in the calculations of limit of detection and supervised Lisa Raoul. Emma Sierecki and Yann Gambin directed the experiments and wrote the manuscript.

Conflicts of Interest: The authors declare no conflict of interest.

\section{Abbreviations}

\begin{tabular}{|c|c|}
\hline PPI & Protein-Protein Interaction \\
\hline sGFP & superfolder GFP \\
\hline eGFP & enhanced GFP \\
\hline mCherry & monomeric Cherry \\
\hline PCA & Protein Complementation Assays \\
\hline DHFR & Dihydrofolate Reductase \\
\hline nLuc & nanoLuc luciferase \\
\hline $\mathrm{L}$ & large bit of the nanoLuc $18 \mathrm{kDa}$ \\
\hline$S$ & small bit of the nanoLuc $1.3 \mathrm{kDa}$ \\
\hline G & anti-GFP nanobody \\
\hline C & anti-Cherry nanobody \\
\hline
\end{tabular}




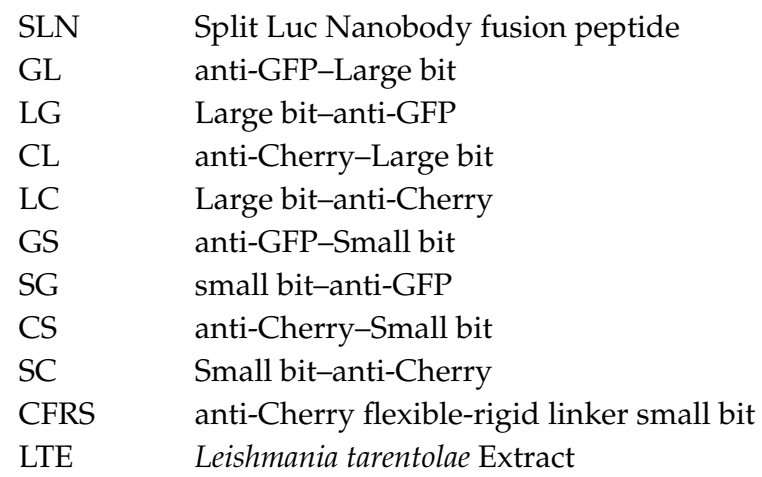

\section{References}

1. Stumpf, M.P.; Thorne, T.; de Silva, E.; Stewart, R.; An, H.J.; Lappe, M.; Wiuf, C. Estimating the size of the human interactome. Proc. Natl. Acad. Sci. USA 2008, 105, 6959-6964. [CrossRef] [PubMed]

2. Wells, J.A.; McClendon, C.L. Reaching for high-hanging fruit in drug discovery at protein-protein interfaces. Nature 2007, 450, 1001-1009. [CrossRef] [PubMed]

3. Braisted, A.C.; Oslob, J.D.; Delano, W.L.; Hyde, J.; McDowell, R.S.; Waal, N.; Yu, C.; Arkin, M.R.; Raimundo, B.C. Discovery of a potent small molecule IL-2 inhibitor through fragment assembly. J. Am. Chem. Soc. 2003, 125, 3714-3715. [CrossRef] [PubMed]

4. Chin, J.W.; Schepartz, A. Design and evolution of a miniature Bcl-2 binding protein. Angew. Chem. Int. Ed. 2001, 40, 3806-3809. [CrossRef]

5. Ding, K.; Lu, Y.; Nikolovska-Coleska, Z.; Qiu, S.; Ding, Y.; Gao, W.; Stuckey, J.; Krajewski, K.; Roller, P.P.; Tomita, Y. Structure-based design of potent non-peptide MDM2 inhibitors. J. Am. Chem. Soc. 2005, 127, 10130-10131. [CrossRef] [PubMed]

6. Rajagopal, S.; Meyer, S.C.; Goldman, A.; Zhou, M.; Ghosh, I. A minimalist approach toward protein recognition by epitope transfer from functionally evolved $\beta$-sheet surfaces. J. Am. Chem. Soc. 2006, 128, 14356-14363. [CrossRef] [PubMed]

7. Engvall, E.; Perlmann, P. Enzyme-linked immunosorbent assay, ELISA. J. Immunol. 1972, 109, 129-135. [PubMed]

8. Homola, J.; Yee, S.S.; Gauglitz, G. Surface plasmon resonance sensors. Sens. Actuators B Chem. 1999, 54, 3-15. [CrossRef]

9. Lea, W.A.; Simeonov, A. Fluorescence Polarization Assays in Small Molecule Screening. Expert Opin. Drug Discov. 2011, 6, 17-32. [CrossRef] [PubMed]

10. Fields, S.; Song, O.-K. A novel genetic system to detect protein-protein interactions. Nature 1989, 340, $245-246$. [CrossRef] [PubMed]

11. Fields, S. High-throughput two-hybrid analysis. FEBS J. 2005, 272, 5391-5399. [CrossRef] [PubMed]

12. Michnick, S.W.; Ear, P.H.; Manderson, E.N.; Remy, I.; Stefan, E. Universal strategies in research and drug discovery based on protein-fragment complementation assays. Nat. Rev. Drug Discov. 2007, 6, 569-582. [CrossRef] [PubMed]

13. Richards, F.M.; Vithayathil, P.J. The preparation of subtilisin-modified ribonuclease and the separation of the peptide and protein components. J. Biol. Chem. 1959, 234, 1459-1465. [PubMed]

14. Johnsson, N.; Varshavsky, A. Split ubiquitin as a sensor of protein interactions in vivo. Proc. Natl. Acad. Sci. USA 1994, 91, 10340-10344. [CrossRef] [PubMed]

15. Ghosh, I.; Hamilton, A.D.; Regan, L. Antiparallel leucine zipper-directed protein reassembly: Application to the green fluorescent protein. J. Am. Chem. Soc. 2000, 122, 5658-5659. [CrossRef]

16. MacDonald, M.L.; Lamerdin, J.; Owens, S.; Keon, B.H.; Bilter, G.K.; Shang, Z.; Huang, Z.; Yu, H.; Dias, J.; Minami, T. Identifying off-target effects and hidden phenotypes of drugs in human cells. Nat. Chem. Biol. 2006, 2, 329-337. [CrossRef] [PubMed]

17. Jach, G.; Pesch, M.; Richter, K.; Frings, S.; Uhrig, J.F. An improved mRFP1 adds red to bimolecular fluorescence complementation. Nat. Methods 2006, 3, 597-600. [CrossRef] [PubMed] 
18. Rossi, F.; Charlton, C.A.; Blau, H.M. Monitoring protein-protein interactions in intact eukaryotic cells by $\beta$-galactosidase complementation. Proc. Natl. Acad. Sci. USA 1997, 94, 8405-8410. [CrossRef] [PubMed]

19. Pelletier, J.N.; Campbell-Valois, F.-X.; Michnick, S.W. Oligomerization domain-directed reassembly of active dihydrofolate reductase from rationally designed fragments. Proc. Natl. Acad. Sci. USA 1998, 95, 12141-12146. [CrossRef] [PubMed]

20. Galarneau, A.; Primeau, M.; Trudeau, L.-E.; Michnick, S.W. $\beta$-Lactamase protein fragment complementation assays as in vivo and in vitro sensors of protein-protein interactions. Nat. Biotechnol. 2002, 20, 619-622. [CrossRef] [PubMed]

21. Luker, K.E.; Smith, M.C.; Luker, G.D.; Gammon, S.T.; Piwnica-Worms, H.; Piwnica-Worms, D. Kinetics of regulated protein-protein interactions revealed with firefly luciferase complementation imaging in cells and living animals. Proc. Natl. Acad. Sci. USA 2004, 101, 12288-12293. [CrossRef] [PubMed]

22. Paulmurugan, R.; Gambhir, S. Monitoring protein-protein interactions using split synthetic renilla luciferase protein-fragment-assisted complementation. Anal. Chem. 2003, 75, 1584-1589. [CrossRef] [PubMed]

23. Massoud, T.F.; Paulmurugan, R.; Gambhir, S.S. A molecularly engineered split reporter for imaging protein-protein interactions with positron emission tomography. Nat. Med. 2010, 16, 921-926. [CrossRef] [PubMed]

24. Müller, M.M.; Kries, H.; Csuhai, E.; Kast, P.; Hilvert, D. Design, selection, and characterization of a split chorismate mutase. Protein Sci. 2010, 19, 1000-1010. [CrossRef] [PubMed]

25. Remy, I.; Michnick, S.W. Application of protein-fragment complementation assays in cell biology. Biotechniques 2007, 42, 137-145. [CrossRef] [PubMed]

26. Shyu, Y.J.; Liu, H.; Deng, X.; Hu, C. Identification of new fluorescent protein fragments for biomolecular fluorescence complementation analysis under physiological conditions. Biotechniques 2006, 40, 61-66. [CrossRef] [PubMed]

27. Ooi, A.T.; Stains, C.I.; Ghosh, I.; Segal, D.J. Sequence-enabled reassembly of $\beta$-lactamase (SEER-LAC): A sensitive method for the detection of double-stranded DNA. Biochemistry 2006, 45, 3620-3625. [CrossRef] [PubMed]

28. Goldberg, A.L. Protein degradation and protection against misfolded or damaged proteins. Nature 2003, 426, 895-899. [CrossRef] [PubMed]

29. Remy, I.; Campbell-Valois, F.; Michnick, S.W. Detection of protein-protein interactions using a simple survival protein-fragment complementation assay based on the enzyme dihydrofolate reductase. Nat. Protoc. 2007, 2, 2120-2125. [CrossRef] [PubMed]

30. Rackham, O.; Brown, C.M. Visualization of RNA-protein interactions in living cells: FMRP and IMP1 interact on mRNAs. EMBO J. 2004, 23, 3346-3355. [CrossRef] [PubMed]

31. Mureev, S.; Kovtun, O.; Nguyen, U.T.; Alexandrov, K. Species-independent translational leaders facilitate cell-free expression. Nat. Biotechnol. 2009, 27, 747-752. [CrossRef] [PubMed]

32. Gambin, Y.; Ariotti, N.; McMahon, K.A.; Bastiani, M.; Sierecki, E.; Kovtun, O.; Polinkovsky, M.E.; Magenau, A.; Jung, W.; Okano, S.; et al. Single-molecule analysis reveals self assembly and nanoscale segregation of two distinct cavin subcomplexes on caveolae. eLife 2014, 3. [CrossRef] [PubMed]

33. Dixon, A.S.; Schwinn, M.K.; Hall, M.P.; Zimmerman, K.; Otto, P.; Lubben, T.H.; Butler, B.L.; Binkowski, B.F.; Machleidt, T.; Kirkland, T.A.; et al. NanoLuc Complementation Reporter Optimized for Accurate Measurement of Protein Interactions in Cells. ACS Chem. Biol. 2016, 11, 400-408. [CrossRef] [PubMed]

34. Kubala, M.H.; Kovtun, O.; Alexandrov, K.; Collins, B.M. Structural and thermodynamic analysis of the GFP:GFP-nanobody complex. Protein Sci. 2010, 19, 2389-2401. [CrossRef] [PubMed]

35. Fridy, P.C.; Li, Y.; Keegan, S.; Thompson, M.K.; Nudelman, I.; Scheid, J.F.; Oeffinger, M.; Nussenzweig, M.C.; Fenyo, D.; Chait, B.T.; et al. A robust pipeline for rapid production of versatile nanobody repertoires. Nat. Methods 2014, 11, 1253-1260. [CrossRef] [PubMed]

36. Sierecki, E.; Stevers, L.M.; Giles, N.; Polinkovsky, M.E.; Moustaqil, M.; Mureev, S.; Johnston, W.A.; Dahmer-Heath, M.; Skalamera, D.; Gonda, T.J.; et al. Rapid mapping of interactions between Human SNX-BAR proteins measured in vitro by AlphaScreen and single-molecule spectroscopy. Mol. Cell. Proteom. 2014, 13, 2233-2245. [CrossRef] [PubMed]

37. Robin, R.; Kainz, B.; Stadlmayr, G.; Schuster, H.; Pum, D.; Messner, P.; Obinger, C.; Schäffer, C. A Fusion Tag to Fold on: The S-Layer Protein SgsE Confers Improved Folding Kinetics to Translationally Fused Enhanced Green Fluorescent Protein. J. Microbiol. Biotechnol. 2012, 22, 1271-1278. 
38. Ve, T.; Vajjhala, P.R.; Hedger, A.; Croll, T.; DiMaio, F.; Horsefield, S.; Yu, X.; Lavrencic, P.; Hassan, Z.; Morgan, G.P.; et al. Structural basis of TIR-domain-assembly formation in MAL- and MyD88-dependent TLR4 signaling. Nat. Struct. Mol. Biol. 2017, 24, 743-751. [CrossRef] [PubMed]

39. Wang, J.; Elahi, A.; Ajidahun, A.; Clark, W.; Hernandez, J.; Achille, A.; Hao, J.-H.; Seto, E.; Shibata, D. The interplay between histone deacetylases and c-Myc in the transcriptional suppression of HPP1 in colon cancer. Cancer Biol. Ther. 2014, 15, 1198-1207. [CrossRef] [PubMed]

40. Coustry, F.; Oh, C.-D.; Hattori, T.; Maity, S.N.; de Crombrugghe, B.; Yasuda, H. The dimerization domain of SOX9 is required for transcription activation of a chondrocyte-specific chromatin DNA template. Nucleic Acids Res. 2010, 38, 6018-6028. [CrossRef] [PubMed]

41. Walhout, A.J.; Gubbels, J.M.; Bernards, R.; van der Vliet, P.C.; Timmers, H.T. c-Myc/Max heterodimers bind cooperatively to the E-box sequences located in the first intron of the rat ornithine decarboxylase (ODC) gene. Nucleic Acids Res. 1997, 25, 1493-1501. [CrossRef] [PubMed]

42. Overman, J.; Fontaine, F.; Moustaqil, M.; Mittal, D.; Sierecki, E.; Sacilotto, N.; Zuegg, J.; Robertson, A.A.B.; Holmes, K.; Salim, A.A.; et al. Pharmacological targeting of the transcription factor SOX18 delays breast cancer in mice. eLife 2017, 6. [CrossRef] [PubMed]

43. Cummins, T.D.; Sapkota, G.P. Characterization of Protein Complexes Using Chemical Cross-Linking Coupled Electrospray Mass Spectrometry. Methods Mol. Biol. 2017. [CrossRef]

44. Grinkevich, P.; Iermak, I.; Luedtke, N.A.; Mesters, J.R.; Ettrich, R.; Ludwig, J. pHluorin-assisted expression, purification, crystallization and X-ray diffraction data analysis of the C-terminal domain of the HsdR subunit of the Escherichia coli type I restriction-modification system EcoR124I. Acta Crystallogr. Sect. F Struct. Biol. Commun. 2016, 72, 672-676. [CrossRef] [PubMed]

45. Hu, J.; Weise, C.; Bottcher, C.; Fan, H.; Yin, J. Expression, purification and structural analysis of functional GABA transporter 1 using the baculovirus expression system. Beilstein J. Org. Chem. 2017, 13, 874-882. [CrossRef] [PubMed]

46. Sjostrand, D.; Diamanti, R.; Lundgren, C.A.K.; Wiseman, B.; Hogbom, M. A rapid expression and purification condition screening protocol for membrane protein structural biology. Protein Sci. Publ. Protein Soc. 2017, 26, 1653-1666. [CrossRef] [PubMed]

47. Unzueta, U.; Seras-Franzoso, J.; Cespedes, M.V.; Saccardo, P.; Cortes, F.; Rueda, F.; Garcia-Fruitos, E.; Ferrer-Miralles, N.; Mangues, R.; Vazquez, E.; et al. Engineering tumor cell targeting in nanoscale amyloidal materials. Nanotechnology 2017, 28. [CrossRef] [PubMed]

48. Bond, T.E.H.; Sorenson, A.E.; Schaeffer, P.M. A green fluorescent protein-based assay for high-throughput ligand-binding studies of a mycobacterial biotin protein ligase. Microbiol. Res. 2017, 205, 35-39. [CrossRef] [PubMed]

49. Garg, H.; Sedano, M.; Plata, G.; Punke, E.B.; Joshi, A. Development of Virus-Like-Particle Vaccine and Reporter Assay for Zika Virus. J. Virol. 2017, 91. [CrossRef] [PubMed]

50. Hwang, G.H.; Park, S.M.; Han, H.J.; Kim, J.S.; Yun, S.P.; Ryu, J.M.; Lee, H.J.; Chang, W.; Lee, S.J.; Choi, J.H.; et al. Purification of small molecule-induced cardiomyocytes from human induced pluripotent stem cells using a reporter system. J. Cell. Physiol. 2017, 232, 3384-3395. [CrossRef] [PubMed]

51. Gambin, Y.; Polinkovsky, M.; Francois, B.; Giles, N.; Bhumkar, A.; Sierecki, E. Confocal Spectroscopy to Study Dimerization, Oligomerization and Aggregation of Proteins: A Practical Guide. Int. J. Mol. Sci. 2016, 17, 655. [CrossRef] [PubMed]

52. Bowes, J.; Brown, A.J.; Hamon, J.; Jarolimek, W.; Sridhar, A.; Waldron, G.; Whitebread, S. Reducing safety-related drug attrition: The use of in vitro pharmacological profiling. Nat. Rev. Drug Discov. 2012, 11, 909-922. [CrossRef] [PubMed]

53. Ross, C.A.; Poirier, M.A. Protein aggregation and neurodegenerative disease. Nat. Med. 2004, 10, S10-S17. [CrossRef] [PubMed]

54. Gagoski, D.; Polinkovsky, M.E.; Mureev, S.; Kunert, A.; Johnston, W.; Gambin, Y.; Alexandrov, K. Performance benchmarking of four cell-free protein expression systems. Biotechnol. Bioeng. 2016, 113, 292-300. [CrossRef] [PubMed]

55. Sierecki, E.; Giles, N.; Bowden, Q.; Polinkovsky, M.E.; Steinbeck, J.; Arrioti, N.; Rahman, D.; Bhumkar, A.; Nicovich, P.R.; Ross, I.; et al. Nanomolar oligomerization and selective co-aggregation of $\alpha$-synuclein pathogenic mutants revealed by single-molecule fluorescence. Sci. Rep. 2016, 6. [CrossRef] [PubMed] 
56. Park, S.; Chung, S.; Kim, K.-M.; Jung, K.-C.; Park, C.; Hahm, E.-R.; Yang, C.-H. Determination of binding constant of transcription factor myc-max/max-max and E-box DNA: The effect of inhibitors on the binding. Biochim. Biophys. Acta Gen. Subj. 2004, 1670, 217-228. [CrossRef] [PubMed]

57. Duff, M.R.; Grubbs, J.; Howell, E.E. Isothermal Titration Calorimetry for Measuring Macromolecule-Ligand Affinity. J. Vis. Exp. 2011. [CrossRef] 